\title{
A Facile Template-Free Room Temperature Synthesis of Mesoporous Wormlike Nickel Phyllosilicate
}

\author{
Farook Adam* and Thiam-Seng Chew
}

School of Chemical Sciences, Universiti Sains Malaysia, 11800 Penang, Malaysia

\begin{abstract}
Mesoporous wormlike nickel phyllosilicate was obtained from rice husk (agricultural biomass) by a simple, facile and template-free method which was carried out via sol-gel technique at room temperature. The synthesized nickel phyllosilicate had been characterized by various physico-chemical methods. Transmission electron micrographs revealed the formation of wormlike network of nickel phyllosilicate with uniform channel width. From the nitrogen sorption analysis, the nickel phyllosilicate shows a high specific BET surface area of $307 \mathrm{~m}^{2} \mathrm{~g}^{-1}$ with a narrow pore size distribution. The amorphous nature of silica and the presence of layered phyllosilicate-like structure were confirmed by x-ray diffraction pattern. The Fourier transform infrared spectrum show the presence of nickel in the silica matrix which was further confirmed by atomic adsorption analysis. The homogeneous dispersion of nickel in the silica matrix was confirmed by energy dispersion X-ray-mapping.
\end{abstract}

Keywords: Phyllosilicate, mesoporous material, wormlike network, rice husk silica, nickel oxide, template-free.

\section{INTRODUCTION}

Since the discovery of M41S by Mobil research group, extensive studies to synthesize mesoporous materials have been done [1,2]. Mesoporous MCM-41 is widely used as catalyst support [3] and adsorbent [4]. This is due to their high surface area, high porosity and well defined pore shape [5]. The major drawback of using MCM-41 is its low thermal and hydrothermal stability [6, 7]. Moreover, MCM-41 possesses one-dimensional hexagonal channels which may be blocked by impurities or other substances [8]. Recently, some scientists have studied the formation of hydrothermally stable wormlike mesoporous material for use in catalysis $[9,10]$ and adsorption studies [11]. These worm like networks can help the diffusion of substances an avoid pore blockage [9].

Various methods have been developed to produce the wormlike structured mesoporous silica [12-14]. Blin et al., [12] used tetramethoxysilane (TMOS) as silica source and decaoxyethylene cetyl ether $\left[\mathrm{C}_{16}(\mathrm{EO})_{10}\right]$ as surfactant (structure directing agent) to produce mesoporous wormlike silica by hydrothermal process. The surfactant was removed by calcination under flow of oxygen at $823 \mathrm{~K}$ for $12 \mathrm{hr}$. Biswas and co-workers [13] synthesized mesoporous wormlike silica by using tetraethylorthosilicate (TEOS) and Tergitol as silica source and template respectively. The researchers used sodium fluoride to polymerize silica and the synthesized product was calcined at $893 \mathrm{~K}$ for $6 \mathrm{hr}$. Park and Pinnavaia [14] reported the formation of mesoporous wormlike silica using commercialized sodium silicate and Jeffamine D2000 as surfactant. The product was obtained by calcination at $827 \mathrm{~K}$ for $4 \mathrm{hr}$ in air. From the economic and environmental point

*Address correspondence to this author at the School of Chemical Sciences, Universiti Sains Malaysia, 11800 Penang, Malaysia; Tel: +6046533567; Fax: +6046574854; E-mails: farook@usm.my, farook_dr@yahoo.com of view, high calcination temperatures to remove the template should be avoided.

Rice husk (RH) is a major waste material from agricultural industry. Open burning of $\mathrm{RH}$ can cause air pollution and breathing problem [15]. Hence, the rice industry face major problem of disposal of RH. RH possesses high content of silica. Some scientists had used $\mathrm{RH}$ as a source of renewable silica [15-17]. The RH silica obtained has high surface area and high porosity [17]. However, pure silica is a neutral framework which limits its application in adsorption and catalysis [9]. Hence, modification of silica networks by incorporating metal have been reported [9, 14]. Xue et al., [18] synthesized $\mathrm{Ni} / \mathrm{SiO}_{2}$ catalyst for hydrogenation of toluene to methyl cyclohexane. Ding and Yan [19] produced nickel catalyst for adsorption of carbon dioxide and methane to study on hydrocarbon transformations.

In this study, the mesoporous wormlike nickel phyllosilicate had been successfully synthesized by a facile templatefree sol-gel preparation. To promote green chemistry, we have used the cheap and renewable RH as silica source for producing the silica-nickel complex.

\section{EXPERIMENTAL PROCEDURE}

\subsection{Raw Materials}

Rice husk (RH) was obtained from a local rice mill in Penang. Nitric acid (QreC. 65\%), sodium hydroxide (QReC, 99\%) and nickel(II) nitrate (Fluka) were used as received. All chemicals are AR grade and used as obtained without further purification.

\subsection{Preparation of Wormlike Nickel Phyllosilicate}

Preparation of sodium silicate was done according to the procedure by Adam and Fua [16]. $30.0 \mathrm{~g}$ of dried RH was 
weighed and transferred into a plastic container. $600 \mathrm{~mL}$ of

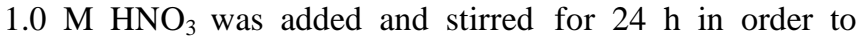
remove unwanted metal. The $\mathrm{RH}$ was filtered and washed with distilled water until it reached a constant $\mathrm{pH}$ (4.5-5.0). The washed $\mathrm{RH}$ was dried in oven at $383 \mathrm{~K}$ overnight. The dried RH was stirred in $500 \mathrm{~mL}$ of $1.0 \mathrm{M} \mathrm{NaOH}$ for $24 \mathrm{~h}$. The mixture was suction filtered to obtain dark brown filtrate (sodium silicate) which was kept in a covered plastic container. Then, the prepared sodium silicate was titrated slowly with a solution of $\mathrm{Ni}\left(\mathrm{NO}_{3}\right)_{2}$ in $3.0 \mathrm{M} \mathrm{HNO}_{3}$ until it reached $\mathrm{pH}$ 9. The obtained colloidal gel was aged in a covered container for two days. The prepared gel was recovered by centrifuge (Zentrifugen Hettich) at $4000 \mathrm{rpm}$, suction filtered and finally followed by washing with distilled water. It was dried in an oven at $383 \mathrm{~K}$ for $24 \mathrm{~h}$. It was allowed to cool in a desiccator. Finally, the product was ground into fine powder and labeled as nickel phyllosilicate. The scheme of preparation of mesoporous wormlike nickel phyllosilicate is shown in Fig. (1).

\section{CHARACTERIZATION OF RH-SILICA/NICKEL}

The nickel phyllosilicate was examined by FTIR in the range of 4000-400 $\mathrm{cm}^{-1}$ (Fig. 2). The adsorption band around 1045,794 and $476 \mathrm{~cm}^{-1}$ are due to $\mathrm{Si}-\mathrm{O}-\mathrm{Si}$ bending and stretching vibrations. The bending vibration of the trapped water molecules in the silica matrix was detected at 1628 $\mathrm{cm}^{-1}$. While, the presence of a broad band at $\sim 3448 \mathrm{~cm}^{-1}$ is due to $\mathrm{O}-\mathrm{H}$ stretching vibration of the silanol or adsorbed water molecules on the silica surface. Adsorption band observed at ca. $667 \mathrm{~cm}^{-1}$ indicates the Ni-O bending vibration [20]. This is a reasonable evidence for the successful incorporation of nickel into the silica matrix. No such band was observed in pure silica.

Powder XRD pattern of nickel phyllosilicate is shown in Fig. (3). It shows a broad peak at $2 \theta=26^{\circ}$ which indicate the amorphous nature of the silica [15]. While the presence of diffraction bands at $2 \theta=35^{\circ}$ and $2 \theta=60^{\circ}$ are due to the

$\mathrm{RH}$ was treated with $1 \mathrm{M} \mathrm{HNO}_{3}$ and $1 \mathrm{M} \mathrm{NaOH}$ in order to get sodium silicate.

The sodium silicate was titrated with nickel nitrate and $3 \mathrm{M} \mathrm{HNO}_{3}$ till $\mathrm{pH}$ 9. Then, ageing the gel formed for 2 days.

Recovered the gel by centrifuge and dried in oven for overnight.

Finally, hand-ground the product and labelled as nickel phyllosilicate.

Fig. (1). The flow diagram of mesoporous wormlike nickel phyllosilicate synthesis.

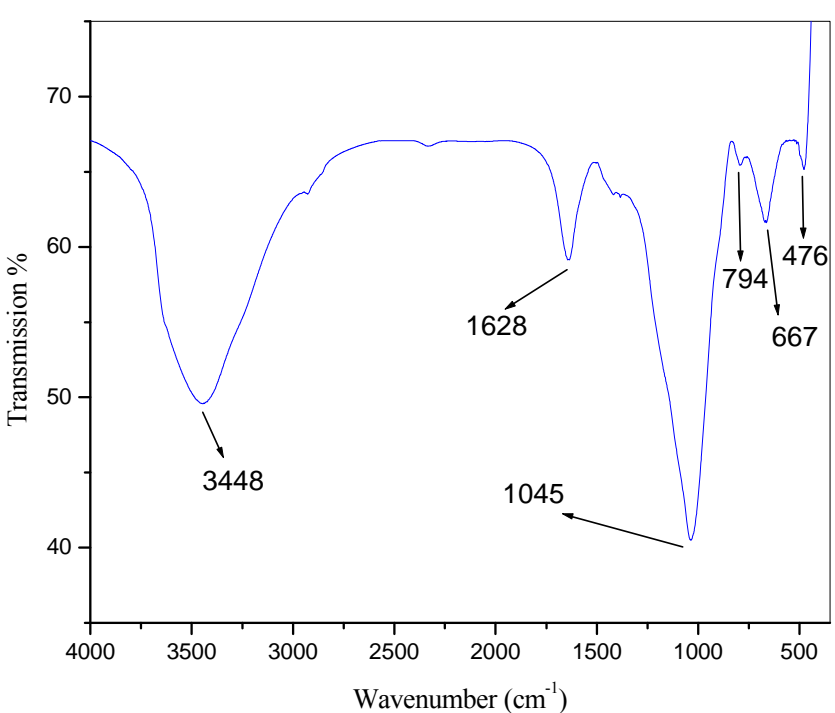

Fig. (2). The FT-IR spectrum of nickel phyllosilicate.

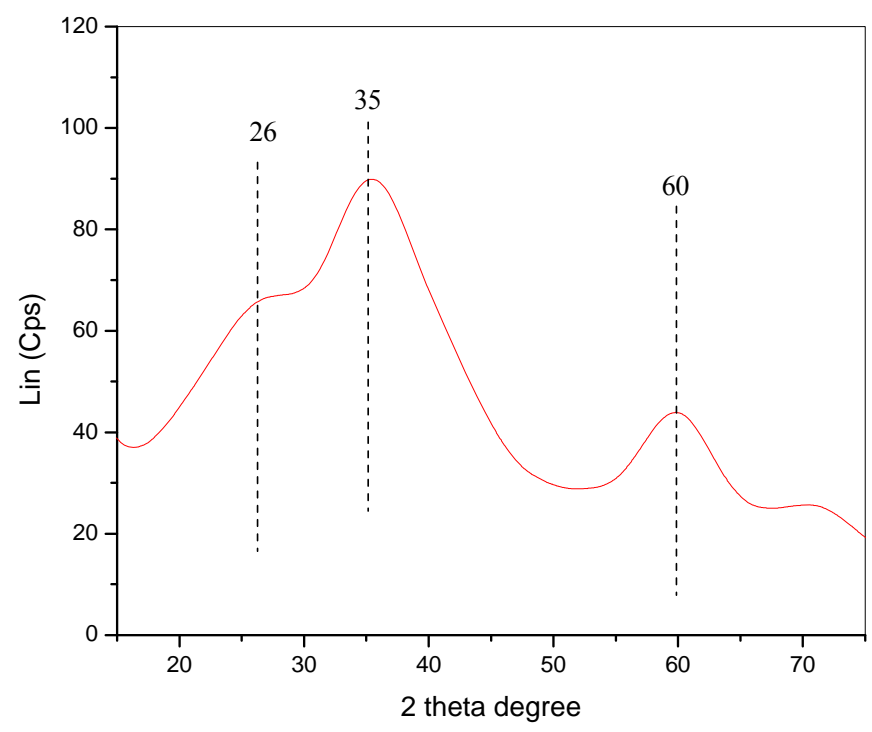

Fig. (3). The X-ray diffractogram of nickel phyllosilicate.

typical layered phyllosilicate-like structure [21]. The absence of crystalline peak indicates the nickel is well dispersed in the silica matrix. This is in accordance with EDX-Mapping result (evidenced later). Due to the disordered nature of the sample (shown in TEM), it is difficult to measure the channel shape and wall thickness by using low-angle XRD pattern (not shown).

$\mathrm{N}_{2}$ adsorption-desorption isotherm of the nickel phyllosilicate is shown in Fig. (4). The sample exhibits type IV isotherm and $\mathrm{H} 3$ hysteresis loop according to IUPAC classification [22]. H3 hysteresis loop is a characteristic of typical mesoporous material consisting of slit-shape capillaries. This is in accordance with TEM result (evidenced later). The sample possesses BET specific surface area of $307 \mathrm{~m}^{2} \mathrm{~g}^{-1}$ and pore volume of $0.16 \mathrm{ccg}^{-1}$. It shows a narrow pore size distribution (PSD) centered at $3.6 \mathrm{~nm}$ derived from BJH model. 
The pore structures of nickel phyllosilicate was examined by transmission electron microscope and is shown in Fig. (5). The nickel phyllosilicate consists of uniform channels while the channels are fully disordered forming wormlike networks. The formation of the wormlike structure may be due to the electrostatic interaction between nickel ion and negatively charged silicate in basic condition [23].

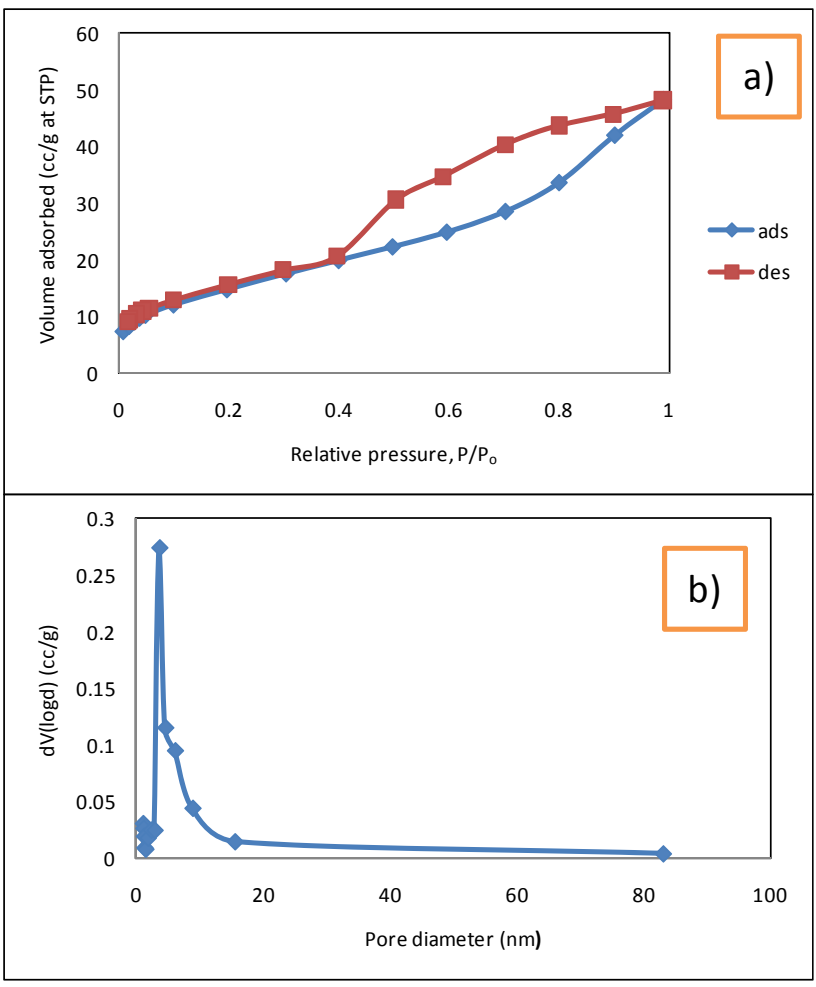

Fig. (4). The surface area analysis of the nickel phylosilicate. a) The nitrogen sorption isotherm of nickel phyllosilicate and b) its pore size distribution.

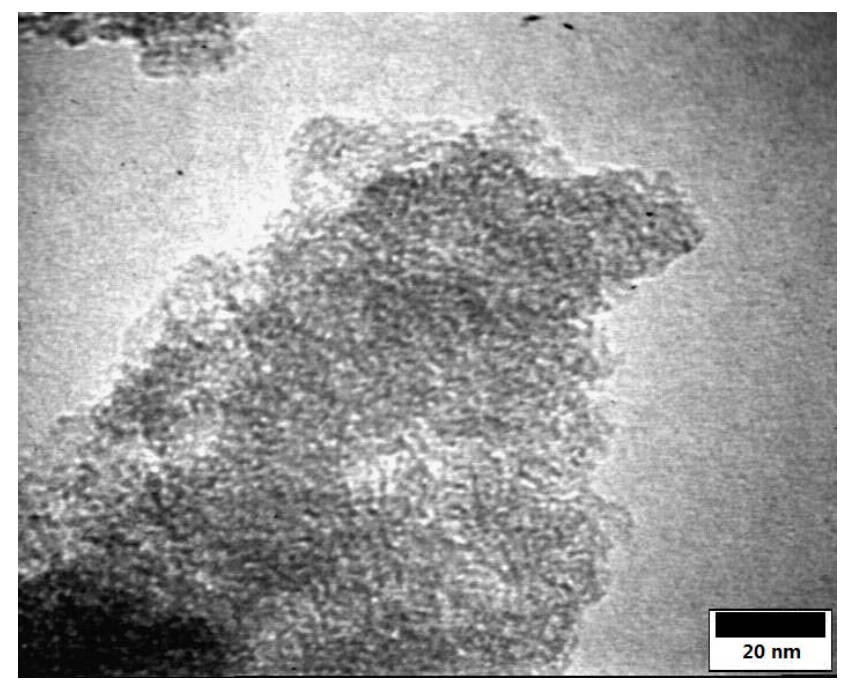

Fig. (5). The TEM micrograph of nickel phyllosilicate at $1050 \mathrm{k}$ magnification showing the wormlike morphology.

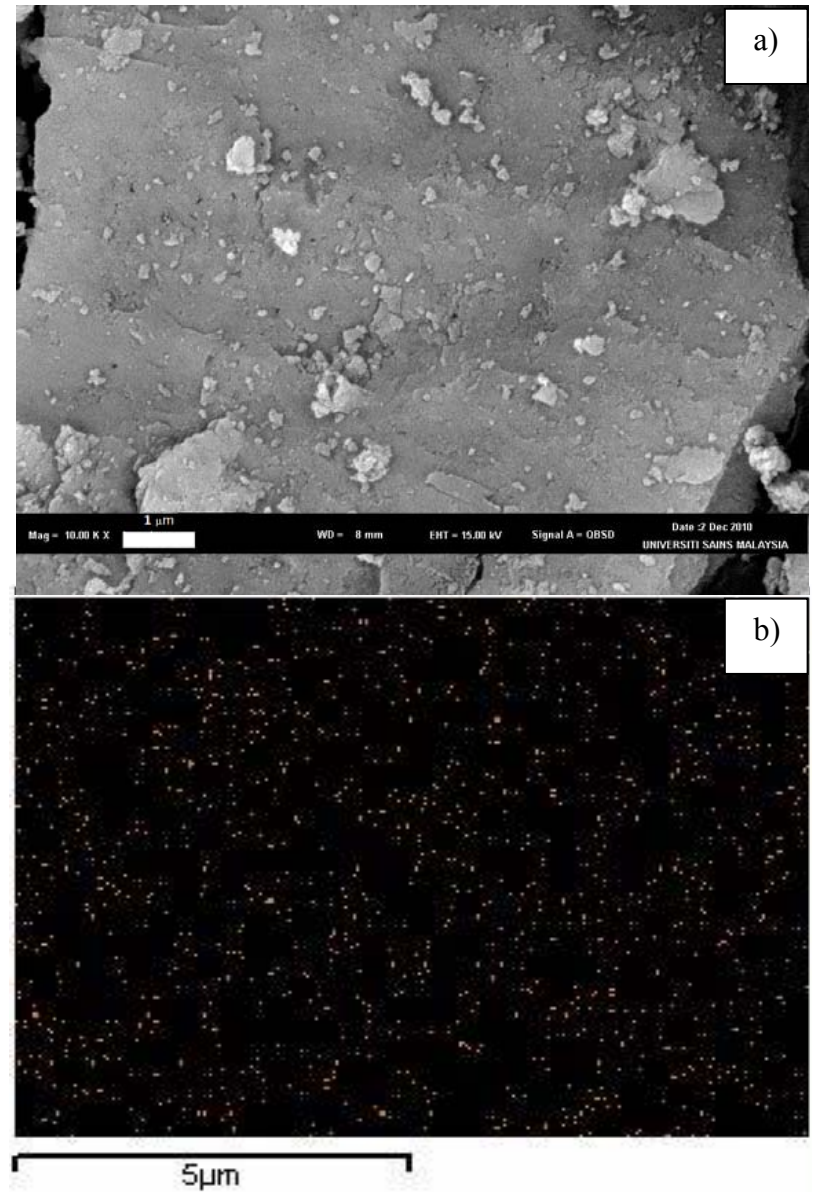

Fig. (6). a) The scanning electron micrograph of nickel phyllosilicate at magnification $10 \mathrm{k}$ and $\mathbf{b}$ ) the energy dispersion X-raymapping for nickel particles on the phylosilicate.

The surface analysis for the nickel phyllosilicate was investigated by scanning electron microscope (SEM) and is shown in Fig. (6a). SEM shows that the complex has a well formed smooth surface. The chemical composition of the nickel phyllosilicate was determined by energy dispersive X-ray (EDX)-mapping. The EDX-mapping (Fig. 6b) shows the presence of nickel particles homogeneously dispersed. To further confirm, atomic adsorption analysis (AAS) was carried for the nickel phyllosilicate. This analysis shows an average nickel loading of $7.7 \mathrm{wt} . \%$.

\section{CONCLUSION}

In this study, an attempt has been made to synthesize wormlike nickel phyllosilicate using $\mathrm{RH}$ as a renewable silica source. The room temperature solvent extraction and sol-gel method was used to obtain nickel phyllosilicate with high surface area and narrow pore size distribution. The room temperature synthesis is certainly very advantageous in terms of energy and cost saving. The mesoporous material shows interconnecting wormlike channels. The synthesis did not involve the use of template molecules. This has avoided the need for calcination which usually requires high temperatures. This is a simple, cheap and low energy method 
to produce wormlike nickel phyllosilicate which will benefit both environmental and industrial needs, with the ever increasing call for greener and safer technology. The method developed is suitable for mass production for wormlike phyllosilicate material and also helps to minimize the disposal problem of RH.

\section{CONFLICT OF INTEREST}

None declared.

\section{ACKNOWLEDGEMENT}

We wish to thank the Government of Malaysia for the RU grant (1001/PKIMIA/811092) which partly supported this work.

\section{REFERENCES}

[1] Bae, J.A.; Song, K.; Jeon, J.; Ko, Y.S.; Park, Y.; Yim, J. Effect of pore structure of amine-functionalized mesoporous silica-supported rhodium catalysts on 1-octene hydroformylation. Micropor. Mesopor. Mater., 2009, 123, 289-297.

[2] Vadia, N.; Rajput, S. Mesoporous material, MCM-41: A new drug carrier. Asian J. Pharm. Clin. Res., 2011, 4, 44-53.

[3] Sakthivel, A.; Selvam, P. Mesoporous (Cr)MCM-41: A mild and efficient heterogeneous catalyst for selective oxidation of cyclohexane. J. Catal., 2002, 211, 134-143.

[4] Lam, K.F.; Fong, C.M.; Yeung, K.L. Separation of precious metals using selective mesoporous adsorbent. Gold Bull., 2007, 40, 192198.

[5] Lelong, G.; Bhattacharyya, S.; Klive, S.; Cacciaguerra, T.; Gonzalez, M.A.; Sabougi, M. Effect of surfactant concentration on the morphology and texture of MCM-41 materials. J. Phys. Chem. C, 2008, 112, 10674-10680.

[6] Ryoo, R.; Kim, J.M.; Ko, K.H.; Shin, C.H. Disordered molecular sieve with branched mesoporous channel network. J. Phys. Chem., 1996, 100, 17718-17721.

[7] Pantazis, C.C.; Trikalitis, P.N.; Pomonis, P.J. Highly loaded and thermally stable $\mathrm{Cu}$-containing mesoporous silica active catalyst for the NO+CO reaction. J. Phys. Chem., 2005, 109, 12574-12581.

[8] Yue, Y.; Sun, Y.; Gao, Z. Disordered mesoporous KIT-1 as a support for hydrodesulfurization catalyst. Catal. Lett., 1997, 47, $167-171$.
[9] Liu, H.; Wang, Z.; Hu, H.; Liang, Y.; Wang, M. Synthesis and characterization of Cr-MSU-1 and its catalytic application for oxidation of styrene. J. Solid State Chem., 2009, 182, 1726-1732.

[10] Xia, Y.; Dai, H.; Jiang, H.; Zhang, L.; Deng, J.; Liu, X. Threedimensionally ordered and wormhole-like mesoporous iron oxide catalysts highly active for the oxidation of acetone and methanol. $J$. Hazard. Mater., 2011, 186, 84-91.

[11] Wang, X.; Li, H.; Liu, H.; Hou, X. AS-synthesized mesoporous silica MSU-1 modified with tetraethylenepentamine for $\mathrm{CO}_{2}$ adsorption. Micropor. Mesopor. Mater. 2011, 142, 564-569.

[12] Blin, J.L.; Leonard, A.; Su, B.L. Synthesis of large pore disordered MSU-type mesoporous silicas through the assembly of $\mathrm{C}_{16}(\mathrm{EO})_{10}$ surfactant and TMOS silica source: effect of the hydrothermal treatment and thermal stability of materials. J. Phys. Chem. B., 2001, 105, 6070-6079.

[13] Biswas, K.; Ray, J.C.; Choi, J.S.; Ahn, W.S. Morphology control of MSU-1 silica particles. J. Non. Cryst. Solid, 2008, 354, 1-9.

[14] Park, I.; Pinnavaia, T.J. Large-pore mesoporous silica with threedimensional wormhole framework structures. Micropor. Mesopor. Mater., 2009, 118, 239-244.

[15] Adam, F.; Chew, T.S; Andas, J. A simple template-free sol-gel synthesis of spherical nanosilica from agricultural biomass. J. SolGel Sci. Technol., 2011, 59,580-583.

[16] Adam, F.; Fua, H.K. Malaysian Patent No: MY-136715-A, 2008.

[17] Foo, K.Y.; Hameed, B.H. Utilization of rice husk ash as novel adsorbent: A judicious recycling of the colloidal agricultural waste. Adv. Colloid Interface Sci., 2009, 152, 39-47.

[18] Xue, M.; Hu, S.; Chen, H.; Fu, Y.; Shen, J. Preparation of highly loaded and dispersed $\mathrm{Ni} / \mathrm{SiO}$ catalysts. Catal. comm., 2011, 12 , 332-336.

[19] Ding, R.G.; Yan, Z.F. Adsorption properties studies of the nickel catalysts for carbon dioxide reforming of methane. Fuel Chem. Div. Preprints, 2002, 46, 103-105.

[20] Parler, C.M.; Ritter, J.A.; Amiridis, M.D. Infrared spectroscopic study of sol-gel derived mixed-metal oxides. J. Non Cryst. solid, 2001, 279, 119-125.

[21] Carta, D.; Loche, D.; Mountjoy, G.; Navarra, G.; Corrias, A. $\mathrm{NiFeO}$ nanoparticles dispersed in an aerogel silica matrix: An Xray absorption study. J. Phys. Chem. C, 2008, 112, 15623-15630.

[22] Chiang, Y.C.; Chiang, P.C.; Chang, E.E. Comprehensive approach to determining the physical properties of granular activated carbons. Chemosphere, 1998, 37, 237-247.

[23] Feltes, T.E.; Espinosa-Alonso, L.; Smit, E.; D’Souza, L.; Meyer R.J.; Weckhuysen, B.M.; REgalbuto, J.R. Selective adsorption of manganese onto cobalt for optimized $\mathrm{Mn} / \mathrm{Co} / \mathrm{TiO}$ Fischer-Tropsch catalysts. J. Catal., 2010, 270, 95-102.

(c) Adam and Chew; Licensee Bentham Open.

This is an open access article licensed under the terms of the Creative Commons Attribution Non-Commercial License (http://creativecommons.org/licenses/by-nc/3.0/) which permits unrestricted, non-commercial use, distribution and reproduction in any medium, provided the work is properly cited. 